\title{
HE4-a biomarker and target in renal fibrosis
}

Previous studies have suggested a possible role for myofibroblasts in the pathogenesis of renal fibrosis. Using a genetic approach to track and isolate myofibroblasts in fibrotic mouse kidneys, researchers have now identified $\mathrm{HE} 4$ - a protease inhibitor that is upregulated in fibrotic kidneys - as a potential biomarker of renal fibrosis and a potential target to inhibit renal fibrosis.

To assess the role of myofibroblastassociated factors in renal fibrosis, Valerie LeBleu and colleagues generated mice that express a fluorescent reporter under the control of the a smooth muscle actin ( (SMA) promoter. They found that the number of interstitial cells expressing aSMA was substantially greater in fibrotic kidneys than in nonfibrotic kidneys. Using gene expression profiling, the researchers identified $\mathrm{He} 4$ (also known as $W f d c 2$ ) as the most upregulated gene in fibrosis-associated myofibroblasts.

The HE4 gene encodes a putative serine protease inhibitor, prompting the researchers to investigate the ability of HE4 to suppress protease activity. Serine protease activity in fibrotic kidney lysates was reduced by preincubation with recombinant HE4. Gene expression profiling of fibrosisassociated fibroblasts and fibrotic mouse kidneys identified two serine proteases with unknown roles in renal fibrosisPrss35 and Prss23. HE4 inhibited Prss35 and Prss 23 serine protease activity and the ability of these serine proteases to degrade type I collagen. The ability of HE4 to inhibit Prss 35 and Prss23 serine protease activity was attenuated by an HE4-neutralizing antibody.

To investigate the functional role of HE4 in renal fibrosis, LeBleu et al. administered an HE4-neutralizing antibody to mice after unilateral ureteral obstruction. Mice that received the antibody had reduced renal fibrosis compared with mice that received a control antibody. Similar outcomes were observed in two other mouse models of renal disease.

HE4, PRSS35 and PRSS23 levels were upregulated in human fibrotic kidneys. In addition, patients with chronic kidney disease and renal fibrosis had elevated serum concentrations of HE4. The researchers suggest that HE4 may therefore serve as a biomarker to predict fibrosis, but caution that large-scale patient analyses are warranted to validate their preliminary findings.

Susan J. Allison

Original article: LeBleu, V. S. et al. Identification of human epididymis protein-4 as a fibroblast-derived mediator of fibrosis. Nat. Med. doi:10.1038/nm.2989 